\title{
Endobronchial ultrasound-guided transbronchial needle aspiration for identifying EGFR mutations
}

\author{
I. Garcia-Olivé*,\#, E. Monsó*,", F. Andreo*,', J. Sanz-Santos*, \\ M. Taron ${ }^{+}$, M.A. Molina-Vila ${ }^{+}$, M. Llatjós ${ }^{\uparrow, \S}$, E. Castellà ${ }^{\uparrow, \S}$, T. Moran ${ }^{+}$, \\ J. Bertran-Alamillo ${ }^{+}$, C. Mayo-de-las-Casas ${ }^{+}$, C. Queralt ${ }^{+}$and R. Rosell
}

ABSTRACT: The presence of somatic mutations of the tyrosine kinase domain of the epidermal growth factor receptor (EGFR) gene in patients with advanced nonsmall cell lung cancer (NSCLC) correlates with a good response to tyrosine kinase inhibitors. The usefulness of endobronchial ultrasound-guided transbronchial needle aspiration (EBUS-TBNA) for the detection of EGFR mutations in cells recovered from malignant mediastinal nodes in patients with NSCLC was assessed.

All patients with lung adenocarcinoma or unspecified NSCLC referred for staging with EBUSTBNA were included. Nodes with a short-axis diameter of $>5 \mathrm{~mm}$ were sampled, and genomic DNA from metastatic tumour cells was obtained for analysis of exons 19 and 21 . The impact of sampling on management was assessed.

EGFR gene analysis of the EBUS-TBNA sample was feasible in 26 (72.2\%) out of the 36 patients with lymph node metastasis. Somatic mutations of the EGFR gene were detected in tissue obtained through EBUS-TBNA in two (10\%) out of 20 patients with metastasic lung adenocarcinoma.

Malignant tissue samples obtained by EBUS-TBNA from patients with nodal metastasis of NSCLC are suitable for the detection of EGFR mutations in most cases, and this technique demonstrates mutated neoplastic cells in a tenth of patients with adenocarcinoma.

KEYWORDS: Adenocarcinoma, endobronchial ultrasonography, endobronchial ultrasonography needle aspiration, epidermal growth factor receptor, lung cancer

$\mathrm{n}$ nonsmall cell lung cancer (NSCLC), the presence of somatic mutations in the part of the gene encoding the tyrosine kinase (TK) domain of the epidermal growth factor receptor (EGFR) correlates with a good response to TK inhibitors, such as gefitinib [1-9] or erlotinib [9-12]. These mutations consist of small in-frame deletions or substitutions clustered around the adenosinetriphosphate-binding site in exons 18, 19 and 21 of the EGFR gene. In most of the reports that have indicated the presence of these mutations, tissue samples had been obtained surgically $[6,7]$. At the moment of diagnosis, however, advanced staging precludes the use of surgery as a first therapeutic approach in a large number of patients, limiting the availability of tissue samples for this kind of analysis. Nonsurgical approaches to tissue sampling are required in order to increase the proportion of cases in which tumour tissue is available for genetic examination. $\mathrm{SHIH}$ et al. [8] reported that the presence of EGFR mutations in needle aspiration samples of lung tumours correlates with a better outcome in these patients, and other studies have since demonstrated the feasibility of transbronchial needle aspiration (TBNA) of tumours reaching the mediastinum or located near the main bronchus in order to obtain samples for mutation studies [13]. More recently, NAKAJIMA et al. [14] demonstrated that EGFR mutations could be detected in samples from metastatic lymph nodes obtained using endobronchial ultrasound (EBUS)-guided TBNA (EBUS-TBNA) in 43 patients suffering from lung cancer. This technique permits the bronchoscopist to perform multiple nonsurgical TBNAs, which give appropriate cytology samples and histology cores in most cases, and has been shown to be useful and safe for both the diagnosis and staging of lung cancer $[15,16]$.

In the present study, the usefulness of EBUSTBNA for obtaining tissue samples from mediastinal and lobar nodes for the analysis of EGFR gene mutations was investigated in all patients

\section{AFFILIATIONS}

*Respiratory Service, Hospital Universitari Germans Trias i Pujol,

${ }^{+}$Medical Oncology Service, Institut Català d'Oncologia, Hospital Universitari Germans Trias i Pujol, ${ }^{\S}$ Pathology Service, Hospital Universitari Germans Trias i Pujol, Badalona,

"Ciber de Enfermedades

Respiratorias, Bunyola, and

\#Dept of Medicine, Universitat

Autònoma de Barcelona, Barcelona, Spain.

CORRESPONDENCE

I. Garcia-Olivé

Servei de Pneumologia Hospital Germans Trias i Pujol Carretera de Canyet $s / n$ 08916 Badalona

Spain

E-mail: ignasi.g.olive@gmail.com

Received:

Feb 172009

Accepted after revision:

July 242009

First published online:

July 302009 
referred for diagnosis or staging who had a final diagnosis of lung adenocarcinoma or unspecified NSCLC.

\section{MATERIAL AND METHODS \\ Subjects and diagnostic procedure}

A prospective study was performed, including all patients who were referred to the North Barcelona Lung Cancer Unit (Hospital Germans Trias i Pujol, Badalona, Spain) for lung cancer diagnosis and/or staging by means of EBUS-TBNA between January 2006 and June 2007 and who had a final diagnosis of lung adenocarcinoma or unspecified NSCLC. In all cases, a computed tomography (CT) scan of the lung, mediastinum and upper abdomen was performed using a multidetector-row spiral CT scanner (Marconi M8000; Philips, Best, the Netherlands) in the month prior to the performance of EBUS-TBNA. Nodes seen to have a short-axis diameter of $>10 \mathrm{~mm}$ on CT were considered to be abnormally enlarged, according to the criteria used in this technique [17, 18]. Sputum cytology and white-light bronchoscopy were additionally performed in patients referred for a diagnostic study, which included bronchial aspiration and bronchoalveolar lavage during the endoscopic procedure. When a bronchial tumour was visible, bronchial biopsy specimens were taken. In patients undiagnosed after the endoscopic procedures and whose CT scan showed a peripheral nodular opacity, transthoracic percutaneous needle aspiration was performed in the absence of any contraindication. Those patients without a final diagnosis after the performance of the diagnostic study whose clinical staging suggested limited disease underwent diagnostic/therapeutic surgery. Patients with coagulation disorders were excluded from staging by EBUS-TBNA and were not included in the present study. All patients agreed to participate and the research protocol was approved by the regional ethics committee (Ethics Committee for Clinical Research of the Hospital Germans Trias i Pujol).

\section{EBUS-TBNA}

EBUS was performed under local anaesthesia and sedation, using a flexible bronchoscope (BF-UC160F-OL8; Olympus Optical Co, Tokyo, Japan) with a distal probe capable of producing linear parallel scans of the mediastinal and parabronchial tissues and a working channel suited to the performance of TBNA under direct ultrasound guidance. Local anaesthesia and sedation were achieved using topical lidocaine spray and intravenous midazolam, respectively, in accordance with standard recommendations $[19,20]$. Nodes with a shortaxis diameter of $\geqslant 5 \mathrm{~mm}$ [21] that were detected during the procedure were sampled under direct ultrasound visualisation with a 21-gauge cytology needle specially designed for EBUSTBNA (NA2015X-4022; Olympus Optical Co). The bronchoscopist proceeded from nodes in regions corresponding to N3 disease to regions corresponding to N1 disease. The needle was guided through the bronchoscope channel as far as the tracheal lumen, and then pushed forward from the sheath and inserted into the tracheal or bronchial wall under ultrasound control until the node was reached. Negative pressure was maintained at the proximal end of the catheter while the needle was pushed forward and backward inside the node. The needle was removed after sampling, rapid on-site cytological examination was performed and the remaining material was processed as a histology core. Pathology findings were classified as being extracted from a normal node, when it contained predominantly lymphocytic cells and no neoplastic cells, or from a metastatic node, when recognisable groups of neoplastic cells were found. A second sample was obtained from metastatic nodes and processed as a histology core for genetic analysis. Aspirates containing bronchial or blood cells alone were considered unsatisfactory, as were samples with isolated groups of neoplastic cells insufficient for genetic analysis; in these situations, the procedure was repeated up to three times [22].

\section{Laboratory methods}

The EGFR mutation screening methodology followed in the present study has been described previously [4, 23]. EGFR status was assessed by microdissection of tumour cells from paraffin-embedded or fresh specimens, and could be determined in samples containing as few as eight tumour cells [23]. Briefly, it involves four steps. 1) Direct laser microdissection (Palm, Oberlensheim, Germany) of tumour cells into PCR buffer. The cells were obtained from either paraffin-embedded or fresh specimens. Paraffin-embedded samples and slides were obtained using standard procedures. Fresh specimens were extended over an appropriate slide, fixed in 96\% ethanol and stained with Harris' haematoxylin for 1 min. 2) A first round of PCR amplification for each EGFR exon [19, 21]. 3) Determination of exon 19 EGFR status by PCR amplification with use of a carboxyfluorescein (FAM)-labelled primer, followed by length analysis in an ABI prism 3130 DNA Analyzer (Applied Biosystems, Foster City, CA, USA). 4) Detection of exon 21 point mutations with a 5'-nuclease PCR assay (TaqMan assay; Applied Biosystems) using FAM and VIC minor groove binder (MGB)-labelled probes for the wild-type and mutant sequence, respectively. This method was used for the examination of neoplastic cells from histology cores obtained by EBUS-TBNA, biological fluids and samples obtained by biopsy or surgery, and was complemented by further analysis using nested PCR and sequencing.

\section{Statistical analysis}

Data were introduced into a database and analysed using SPSS software version 15.0 (SPSS, Inc., Chicago, IL, USA). Results are expressed as absolute and relative frequencies for categorical variables and as mean $\pm \mathrm{SD}$ for continuous variables. First, the availability of an adequate tissue sample for genetic examination of metastatic nodes from patients with a diagnosis of lung adenocarcinoma or NSCLC was assessed. Secondly, the prevalences of EGFR mutations in the examined nodal samples of both adenocarcinoma and unspecified NSCLC were determined. Finally, the impact of these results on choice of treatment was assessed.

\section{RESULTS}

\section{Patient characteristics}

Of the 185 patients referred to the North Barcelona Lung Cancer Unit for diagnosis and/or staging during the study period who had a final diagnosis of lung cancer, 40 had adenocarcinoma and 11 had unspecified NSCLC. These patients comprised the study population sample (table 1). CT scan findings in these 51 patients (mean \pm SD age $62.6 \pm 9.1 \mathrm{yrs}$; $41(80.4 \%)$ males) were lung tumour with a long axis of $>30 \mathrm{~mm}$ in $24(47.1 \%)$ patients and $\leqslant 30 \mathrm{~mm}$ in $20(39.2 \%)$, alveolar opacity or atelectasia in six $(11.7 \%)$ and pleural 
effusion in one $(2 \%)$. These patients underwent EBUS-TBNA, and a total number of 70 lymph nodes were identified and sampled using this technique. EBUS revealed lymph nodes in regions $2 R(n=2), 2 L(n=2), 4 R(n=17), 4 L(n=5), 7(n=33)$ and $10 / 11(n=11)$ in these 51 patients, with a median diameter of $11 \mathrm{~mm}$ (interquartile range (IQR) 9-17 mm). Examination of EBUS-TBNA samples from the observed nodes showed lung cancer cells in one or more samples in 36 patients (27 adenocarcinoma and nine unspecified NSCLC) and normal lymph node cells alone in 12 patients. Samples from three patients were nondiagnostic.

In $35(68.6 \%)$ out of the 51 patients studied, it was feasible to perform a genetic study on neoplastic cells recovered by nonsurgical techniques, using either TBNA samples (26 patients) or other cytological specimens (nine patients). EGFR

\section{TABLE 1 Patient characteristics}

\begin{tabular}{lc} 
Subjects $\mathbf{n}$ & 51 \\
Age yrs & $62.6 \pm 9.1$ \\
Female sex & $10(19.6)$ \\
Stage & \\
IIA & $2(3.9)$ \\
IIB & $3(5.9)$ \\
IIIA & $19(37.3)$ \\
IIIB & $9(17.6)$ \\
IV & $18(35.3)$ \\
Diagnosis & \\
Adenocarcinoma & $40(78.4)$ \\
NSCLC & $11(21.6)$ \\
Diagnostic procedure & \\
EBUS-TBNA & $26(51.0)$ \\
Bronchoalveolar lavage & $1(2.0)$ \\
Bronchial biopsy & $5(9.8)$ \\
Pericardial fluid & $1(2.0)$ \\
Bone metastasis & $2(3.9)$ \\
Surgery & $16(31.4)$ \\
Lymph node metastasis by EBUS-TBNA & $36(70.6)$ \\
Adenocarcinoma & $29(56.9)$ \\
Unspecified NSCLC & $7(13.7)$ \\
Metastatic lymph node region sampled & \\
4R & $10(19.6)$ \\
4L & $2(3.9)$ \\
7 & $22(43.1)$ \\
Lobar node & $2(3.9)$ \\
EGFR analysis feasible & \\
Adenocarcinoma EBUS-TBNA samples & $20(39.2)$ \\
NSCLC EBUS-TBNA samples & $6(11.8)$ \\
Other biopsy/cytology samples & $25(49.0)$ \\
EGFR mutation status & \\
Present & $5(9.8)$ \\
$\quad$ Exon 19 & $2(3.9)$ \\
Mild-type & $3(5.9)$ \\
Mutation identified only in EBUS-TBNA sample & $2(3.9)$ \\
\hline
\end{tabular}

Data are presented as mean \pm SD or $n(\%)$, unless otherwise indicated. NSCLC: nonsmall cell lung cancer; EBUS-TBNA: endobronchial ultrasound-guided transbronchial needle aspiration; EGFR: epidermal growth factor receptor mutations were analysed in the TBNA samples obtained from $26(72.2 \%)$ patients with metastatic nodal specimens demonstrated by EBUS-TBNA, 20 with adenocarcinoma and six with unspecified NSCLC. In the remaining 10 node-positive patients, no additional tumour cells were available for genetic analysis in the EBUS-TBNA specimens after examination for diagnosis and repetition of the procedure up to three times. The nine nonsurgical samples with adenocarcinoma or unspecified NSCLC cells suitable for genetic analysis in the series were bronchial biopsy specimens (five cases; $9.8 \%$ ), bronchoalveolar lavage fluid (one case; $2 \%$ ), pericardial fluid (one case; $2 \%$ ) and needle aspirations of a bone metastasis (two cases; 3.9\%). In 16 $(31.4 \%)$ patients without a final diagnosis after the performance of nonsurgical techniques whose clinical staging following EBUS-TBNA suggested local disease, the final diagnosis of adenocarcinoma or unspecified NSCLC was attained after therapeutic surgery. On surgery, mediastinal and hilar lymph nodes were normal in 12 of these cases, but N2/N1 disease was found in single nodes out of reach of EBUS-TBNA in four patients. In these 16 patients, the genetic studies were performed using the surgically obtained lung tumour tissue.

\section{EGFR mutation}

Lung adenocarcinoma was the diagnosis for 40 patients in the present study, and analysis of the EGFR in these patients found mutations in five $(12.5 \%)$ of them, two cases in exon 19 and three in exon 21. Histology cores obtained using EBUSTBNA were the only samples permitting demonstration of an EGFR mutation in two (5\%) patients. In the three additional adenocarcinoma patients who exhibited EGFR mutations, the abnormality was identified in cells recovered from a malignant pericardial effusion (one case) and in tumour samples obtained during the surgical procedure (two cases). No lymph node metastasis was demonstrated by EBUS-TBNA in these three patients. No EGFR mutations were identified in the 11 patients with unspecified NSCLC, neither in the six patients with lymph node metastasis identified on EBUS-TBNA sampling nor in the available samples from the other five patients (table 2). The examination of EGFR mutations in samples from the 40 patients with lung adenocarcinoma, accordingly, led to a change in treatment in the five $(12.5 \%)$ patients who showed an EGFR mutation, and, in two (5\%) of these cases, this change in management depended upon a sample obtained by EBUSTBNA, which was the only one showing the genetic abnormality in the metastatic adenocarcinoma cells. All patients showing an EGFR mutation were treated with erlotinib and were free of disease progression for a median of 26 months (IQR 1836 months).

\section{DISCUSSION}

The objective of the present study was to determine the suitability, for EGFR mutation analysis, of samples of metastatic lymph nodes obtained through EBUS-TBNA from patients with lung adenocarcinoma or unspecified NSCLC. To that end, the prevalence of positive cases in these samples and the impact of the performance of this diagnostic procedure on treatment were determined. EBUS-TBNA permitted the identification of metastasic nodal disease in $36(70.6 \%)$ out of the 51 patients with these types of lung cancer, and the samples recovered using this technique were appropriate for genetic examination of EGFR mutations in most $(72.2 \%)$ of 


\begin{tabular}{lccccc}
\hline TABLE 2 & $\begin{array}{l}\text { Patients with epidermal growth factor receptor } \\
\text { mutations }\end{array}$ \\
$\begin{array}{l}\text { Patient } \\
\text { No. }\end{array}$ & $\begin{array}{c}\text { Age } \\
\text { yrs }\end{array}$ & Sex & Mutation exon & $\begin{array}{c}\text { Sampling } \\
\text { technique }\end{array}$ & Stage \\
\hline $\mathbf{1}$ & 62 & Male & 21 (Leu858Arg) & Surgery & IV \\
$\mathbf{2}$ & 47 & Female & 21 (Leu858Arg) & Pericardiocentesis & IV \\
$\mathbf{3}$ & 69 & Male & 19 (745-750del) & EBUS-TBNA & IV \\
$\mathbf{4}$ & 70 & Female & 21 (Leu858Arg) & Surgery & IIIA \\
$\mathbf{5}$ & 71 & Male & 19 (751-759del) & EBUS-TBNA & IV \\
\hline
\end{tabular}

In all cases, the patients exhibited an adenocarcinoma pathology. Leu858Arg: substitution of leucine 858 with arginine; del: deletion of codon numbers indicated; EBUS-TBNA: endobronchial ultrasound-guided transbronchial needle aspiration

these cases. Samples obtained by EBUS-TBNA were the only ones confirming the presence of an EGFR mutation in two patients with lung adenocarcinoma $(5.0 \%$ of the 40 patients with this type of lung cancer). The present results confirm the usefulness of EBUS-TBNA for obtaining samples appropriate for genetic analysis in patients with lung cancer.

Since EGFR was initially signalled as an attractive target for cancer chemotherapy [24], reports have shown a relationship between the presence of EGFR mutations and a better response to TK inhibitors in patients with lung adenocarcinoma and unspecified NSCLC [1-12]. Since the 2006, some reports have focused on proving that it is feasible to detect EGFR mutations in nonsurgical specimens, a particularly relevant approach considering that most patients with lung cancer have a disseminated stage of the disease at diagnosis. In these studies, EGFR mutations have been detected in bronchoscopic biopsy and cytology specimens $[25,26]$, percutaneous and pleural biopsy specimens, effusion cell blocks and blood samples [8, 27, 28]. Cases in which EGFR mutations were identified in TBNA and EBUS-TBNA samples have also been reported recently $[13,14]$.

The present results support the study of mutations of the EGFR gene in samples from metastatic nodes obtained through EBUS-TBNA in lung adenocarcinoma. The prevalence of EGFR gene mutations in these patients in the present series is close to $10 \%$, a frequency similar to that reported in another study in Spain that examined lung tumour samples [3]. In the present series, EBUS-TBNA provided the only sample that showed EGFR mutations in 5\% of patients with lung adenocarcinoma, and emerged as a technique with a clear advantage over other nonsurgical techniques for obtaining samples suitable for genetic analysis in these patients. Sampling with EBUSTBNA is performed under direct vision, and, since it is a real-time procedure, can be repeated in order to obtain the volume of tumour cells appropriate for genetic determinations.

Mutations of the EGFR gene were not found in the 11 studied patients with unspecified NSCLC. The reported prevalence of EGFR mutations in this type of lung cancer is lower than that in adenocarcinoma cases. This observation is in line with other studies, in which the greater number of EGFR mutations occurred among those with adenocarcinoma, their prevalence being lower in those with unspecified NSCLC [2, 6, 7, 28, 29]. In some of these previous studies, as in the present one, mutations were, likewise, not found in unspecified NSCLC cases $[7,29,30]$; others, however, have detected mutations in this type of lung cancer $[2,6]$. Thus, despite a probably lower prevalence in unspecified NSCLC, and the negativity of the present results in patients with this diagnosis, the study of EGFR mutations in samples obtained by EBUS-TBNA from patients with unspecified NSCLC may occasionally identify mutations in patients, who can be treated accordingly.

EGFR mutations were found in slightly more than a tenth of the patients with adenocarcinoma in the present study, a figure lower than the $25 \%$ prevalence found by NAKIJAMA et al. [14] in Asians, an expected finding considering the different genetic backgrounds of European and Eastern Asian populations [31, 32]. In the present patients, EGFR mutations in exon 21 were single point mutations at amino acid 858 , as was reported by NAKIJAMA et al. [14], although those authors also found a double point mutation of this exon in one patient. The present results confirm that EBUS-TBNA is a useful technique for obtaining histology cores suitable for genetic analysis in European populations, who show a lower prevalence of these mutations than Asians.

To conclude, the present study demonstrated that samples obtained by EBUS-TBNA are useful for detecting EGFR mutations in patients with lung adenocarcinoma, and positive results can be expected in nearly a tenth of patients with this diagnosis studied using this technique who exhibit metastasis in mediastinal or lobar nodes, EBUS-TBNA being the only technique providing adequate samples for this determination in $5 \%$ of patients. The results obtained by genetic analysis of the material recovered by EBUS-TBNA may determine a change in the treatment pattern of these patients, permitting its simplification and improving their prognosis.

\section{SUPPORT STATEMENT}

This study was supported by funds from the Centre for Biomedical Research in Respiratory Diseases (CibeRes; Bunyola, Spain), Health Research Fund (Madrid, Spain; 070170), Spanish Society of Pulmonology and Thoracic Surgery (Barcelona, Spain), Catalan Pulmonology Society (Barcelona, Spain), Spanish Respiratory Endoscopy Association (Seville, Spain) and Thematic Network of Cooperative Cancer Research (Salamanca, Spain; RD06/0020/0056). The CibeRes is an initiative of the Carlos III Health Institute (Madrid, Spain).

\section{STATEMENT OF INTEREST}

None declared.

\section{ACKNOWLEDGEMENTS}

The authors thank A. Ruiz for technical assistance during the performance of the procedures. M.E. Kerans assisted with English expression in versions of the manuscript.

\section{REFERENCES}

1 Park J, Park BB, Kim JY, et al. Gefitinib (ZD1839) monotherapy as a salvage regimen for previously treated advanced non-small cell lung cancer. Clin Cancer Res 2004; 10: 4383-4388.

2 Lynch TJ, Bell DW, Sordella R, et al. Activating mutations in the epidermal growth factor receptor underlying responsiveness of 
non-small-cell lung cancer to gefitinib. N Engl J Med 2004; 350: 2129-2139.

3 Cortes-Funes H, Gomez C, Rosell R, et al. Epidermal growth factor receptor activating mutations in Spanish gefitinib-treated nonsmall-cell lung cancer patients. Ann Oncol 2005; 16: 1081-1086.

4 Taron M, Ichinose $\mathrm{Y}$, Rosell $\mathrm{R}$, et al. Activating mutations in the tyrosine kinase domain of the epidermal growth factor receptor are associated with improved survival in gefitinib-treated chemorefractory lung adenocarcinomas. Clin Cancer Res 2005; 11: 5878-5885.

5 Rosell R, Ichinose $\mathrm{Y}$, Taron M, et al. Mutations in the tyrosine kinase domain of the EGFR gene associated with gefitinib response in nonsmall-cell lung cancer. Lung Cancer 2005; 50: 25-33.

6 Han SW, Kim TY, Hwang PG, et al. Predictive and prognostic impact of epidermal growth factor receptor mutation in nonsmall-cell lung cancer patients treated with gefitinib. J Clin Oncol 2005; 23: 2493-2501.

7 Mitsudomi T, Kosaka T, Endoh $\mathrm{H}$, et al. Mutations of the epidermal growth factor receptor gene predict prolonged survival after gefitinib treatment in patients with non-small-cell lung cancer. J Clin Oncol, 2005: 2513-2520.

8 Shih J-Y, Gow C-H, Yu C-J, et al. Epidermal growth factor receptor mutations in needle biopsy/aspiration samples predict response to gefitinib therapy and survival of patients with advanced nonsmall cell lung cancer. Int J Cancer 2006; 118: 963-969.

9 Rosell R, Perez-Roca L, Sanchez JJ, et al. Customized treatment in non-small-cell lung cancer based on EGFR mutations and BRCA1 mRNA expression. PLoS One 2009; 4: e5133.

10 Bendedetti G, Latini L, Galetta D, et al. Epidermal growth factor receptor exon 19 deletions predict complete regression of multiple intracranial metastases in two cases of non-small cell lung cancer treated with erlotinib. J Thorac Oncol 2009; 4: 936-937.

11 Kaira K, Naito T, Takahashi T, et al. Pooled analysis of the reports of erlotinib after failure of gefitinib for non-small cell lung cancer. Lung Cancer 2009; [Epub ahead of print DOI: 10.1016/j.lungcan. 2009.05.006].

12 Zhou ZT, Xu XH, Wei Q, et al. Erlotinib in advanced non-small-cell lung cancer after gefitinib failure. Cancer Chemother Pharmacol 2009; 64: 1123-1127.

13 Horiike A, Kimura H, Nishio K, et al. Detection of epidermal growth factor receptor mutation in transbronchial needle aspirates of non-small cell lung cancer. Chest 2007; 131: 1628-1634.

14 Nakajima T, Yasufuku K, Suzuki M, et al. Assessment of epidermal growth factor receptor mutation by endobronchial ultrasoundguided transbronchial needle aspiration. Chest 2007; 132: 597-602.

15 Yasufuku K, Chiyo M, Sekine Y, et al. Real-time endobronchial ultrasound-guided transbronchial needle aspiration of mediastinal and hilar lymph nodes. Chest 2004; 126: 122-128.

16 Yasufuku K, Chiyo M, Koh E, et al. Endobronchial ultrasound guided transbronchial needle aspiration for staging of lung cancer. Lung Cancer 2005; 50: 347-354.

17 Colice GL. Chest CT for known or suspected lung cancer. Chest 1994; 106: 1538-1550.
18 Quint LE, Francis IR, Wahl RL, et al. Preoperative staging of nonsmall cell carcinoma of the lung: imaging methods. AJR Am J Roentgenol 1995; 164: 1349-1359.

19 Reed AP. Preparation of the patient for awake flexible fiberoptic bronchoscopy. Chest 1992; 101: 244-253.

20 British Thoracic Society Bronchoscopy Guidelines Committee, a Subcommittee of the Standards of Care Committee of the British Thoracic Society, British Thoracic Society guidelines on diagnostic flexible bronchoscopy. Thorax 2001; 56: Suppl. 1, 1-21.

21 Garcia-Olivé I, Monsó E, Andreo F, et al. Sensitivity of linear endobronchial ultrasonography and guided transbronchial needle aspiration for the identification of nodal metastasis in lung cancer staging. Ultrasound Med Biol 2009; 35: 1271-1277.

22 Baker JJ, Solanki PH, Schenk DA, et al. Transbronchial fine needle aspiration of the mediastinum. Importance of lymphocytes as an indicator of specimen adequacy. Acta Cytol 1990; 34: 517-523.

23 Molina-Vila MA, Bertran-Alamillo J, Reguart N, et al. A sensitive method for detecting EGFR mutations in non-small cell lung cancer samples with few tumor cells. J Thorac Oncol 2008; 3: 1224-1235.

24 Woodburn JR. The epidermal growth factor receptor and its inhibition in cancer therapy. Pharmacol Ther 1999; 82: 241-250.

25 Fujita S, Mio T, Sonobe M, et al. Accuracy of epidermal growth factor receptor mutation analysis on the basis of small biopsy specimens in patients with nonsmall cell lung cancer. Int J Cancer 2006; 119: 1751-1752.

26 Oshita F, Matsukuma S, Yoshihara M, et al. Novel heteroduplex method using small cytology specimens with a remarkably high success rate for analysing EGFR gene mutations with a significant correlation to gefitinib efficacy in non-small-cell lung cancer. $\mathrm{Br} J$ Cancer 2006; 95: 1070-1075.

27 Kimura H, Fujiwara Y, Sone T, et al. High sensitivity detection of epidermal growth factor receptor mutations in the pleural effusion of non-small cell lung cancer patients. Cancer Sci 2006; 97: 642-648.

28 Kimura H, Kasahara K, Kawaishi M, et al. Detection of epidermal growth factor receptor mutations in serum as a predictor of the response to gefitinib in patients with non-small-cell lung cancer. Clin Cancer Res 2006; 12: 3915-3921.

29 Paez JG, Janne PA, Lee JC, et al. EGFR mutations in lung cancer: correlation with clinical response to gefitinib therapy. Science 2004; 304: 1497-1500.

30 Tokumo M, Toyooka S, Kiura K, et al. The relationship between epidermal growth factor receptor mutations and clinicopathologic features in non-small cell lung cancers. Clin Cancer Res 2005; 11: 1167-1173.

31 Bai H, Mao L, Wang HS, et al. Epidermal growth factor receptor mutations in plasma DNA samples predict tumor response in Chinese patients with stages IIIB to IV non-small-cell lung cancer. J Clin Oncol 2009; 27: 2653-2659.

32 Sasaki H, Shimizu S, Okuda K, et al. Epidermal growth factor receptor gene amplification in surgical resected Japanese lung cancer. Lung Cancer 2009; 64: 295-300. 